\title{
Recurrent Hepatitis C-Related Membranoproliferative Glomerulonephritis in a Simultaneous Liver-Kidney Transplant Recipient With Atypical Presentation and Clinical Course
}

\author{
Mumnoon Haider ${ }^{\mathrm{a}}$, Bruce A. Jones ${ }^{\mathrm{b}}$, K. K. Venkat ${ }^{\mathrm{a}, \mathrm{c}}$
}

\begin{abstract}
Hepatitis $\mathrm{C}$ virus (HCV) infection is known to be associated with membranoproliferative glomerulonephritis (MPGN) with or without coexistent cryoglobulinemia. We report a patient with end-stage liver and renal disease caused by HCV infection (biopsy-proven liver cirrhosis and MPGN, respectively). Approximately 10 months after simultaneous liver-kidney transplantation, patient developed severe acute kidney injury (AKI) with tripling of the baseline serum creatinine level. Allograft biopsy showed recurrent HCV-related cryoglobulinemic MPGN. However, urinalysis done immediately prior to biopsy did not suggest MPGN (lack of proteinuria and microscopic hematuria). Subsequent clinical course was also atypical in that the severe AKI resolved spontaneously and completely over 2 weeks without any change in maintenance post-transplant immunosuppressive medications, or use of plasmapheresis or HCV-antiviral therapy. Three months after resolution of recurrent MPGN-related AKI, institution of interferon-free anti-HCV therapy (sofosbuvir + ribavirin) resulted in clearance of $\mathrm{HCV}$ viremia over 18 weeks while liver and kidney transplant function remained stable/good. This patient illustrates the following: HCV-related MPGN may recur in the kidney transplant without typical urinary findings, and the resultant (even severe) AKI may resolve spontaneously without specific treatment and the excellent outcome that can be achieved with the newer anti-HCV therapies.
\end{abstract}

Keywords: Hepatitis C virus; Liver-kidney transplantation; Recurrent membranoproliferative glomerulonephritis; Atypical presentation of recurrent MPGN; Newer anti-HCV therapies

Manuscript accepted for publication October 17, 2014

aDivision of Nephrology and Transplantation Institute, Henry Ford Hospital, 2799 W Grand Blvd, Detroit, MI 48202, USA

bDepartment of Pathology and Laboratory Medicine, Henry Ford Hospital, 2799 W Grand Blvd, Detroit, MI 48202, USA

${ }^{\mathrm{c}}$ Corresponding Author: K. K. Venkat, Division of Nephrology, Henry Ford Hospital, 2799 W Grand Blvd, CFP 502, Detroit, MI 48202, USA.

Email: kvenkat1@hfhs.org

doi: http://dx.doi.org/10.14740/wjnu185e

\section{Introduction}

The most common renal disease occurring in patients with hepatitis $\mathrm{C}$ virus $(\mathrm{HCV})$ infection is membranoproliferative glomerulonephritis (MPGN). HCV-related MPGN usually occurs in the setting of HCV-related cryoglobulinemia (more common with type 2 (monoclonal IgM-kappa + polyclonal $\operatorname{IgG}+$ viral capsid antigens) than type 3 (polyclonal IgM + polyclonal $\operatorname{IgG}+$ viral capsid antigens) cryoglobulinemia) [1, 2]. However, HCV-related MPGN may also occur in the absence of cryoglobulinemia [2].

The commonest cause of type 2 and type 3 cryoglobulinemia in clinical practice is chronic HCV infection (less commonly hepatitis B virus (HBV) infection) $[3,4]$. Cryoglobulins precipitate at temperatures below $37^{\circ} \mathrm{C}$ and re-dissolve upon warming. Type 2 and type 3 cryoglobulins can activate the complement cascade through the lectin pathway resulting in marked reduction in the serum concentration of $\mathrm{C} 4$ complement with a lesser or no effect on C3 complement level [5]. Type 2 and type 3 cryoglobulins frequently show rheumatoid factor (RF) activity [5]. A positive RF test is often used as a surrogate marker for these types of cryoglobulinemia. Specific testing for cryoglobulins requires blood collection, sample transportation to the laboratory and serum separation all at 37 ${ }^{\circ} \mathrm{C}$ followed by cold-storage at $4{ }^{\circ} \mathrm{C}$ for $5-7$ days before testing for cryoglobulins [6,7]. A positive test for cryoglobulins can be reported as "cryocrit" (percentage of the centrifuged cold serum sample that is cryoprecipitate) or as the quantity of cryoglobulin on immunoelectrophoresis [8]. The latter methodology can separate monoclonal from polyclonal cryoglobulins and identify the type of light chains (kappa, lambda or both) in the cryoglobulins.

Since cryoglobulinemic MPGN is a proliferative (and sometimes necrotizing and crescentic) type of glomerulonephritis, it is typically associated with proteinuria and active urinary sediment (microscopic hematuria \pm RBC casts) [5]. Clinical presentation can vary from asymptomatic urinary abnormalities, acute nephritic syndrome, nephrotic syndrome, mixed nephritic-nephrotic syndrome or rapidly progressive glomerulonephritis [5]. Renal function may be normal, or the patient may present with acute kidney injury (AKI) or chronic progressive renal failure with approximately $10-14 \%$ of patients reaching end-stage renal disease (ESRD) [9]. Although

Articles @ The authors | Journal compilation @ World J Nephrol Urol and Elmer Press Inc ${ }^{\mathrm{TM}} \quad$ | www.wjnu.elmerpress.com

This is an open-access article distributed under the terms of the Creative Commons Attribution License, which permits unrestricted use, distribution, and reproduction in any medium, provided the original work is properly cited 

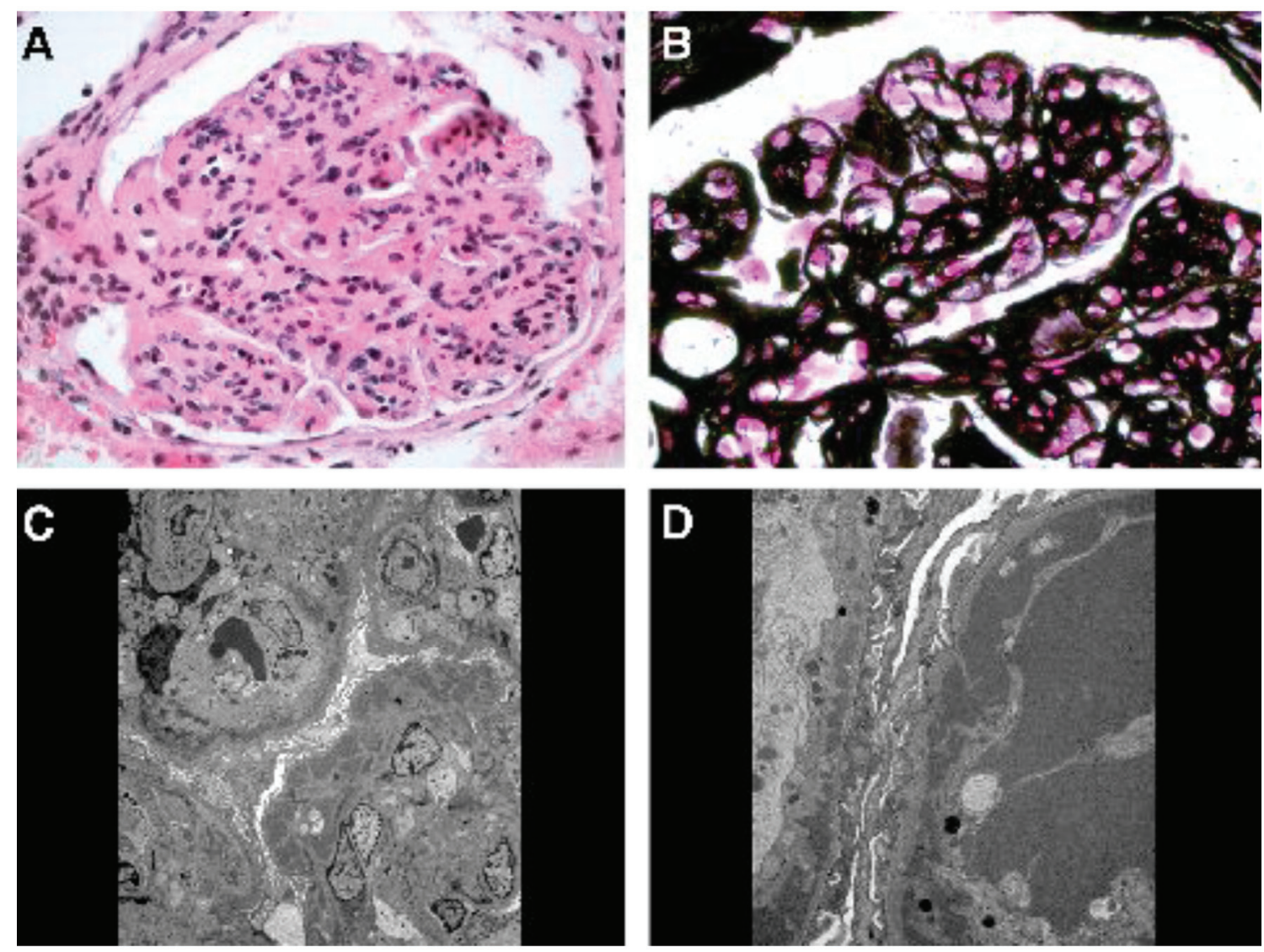

Figure 1. Pre-transplant native renal biopsy. (A) Glomerulus exhibiting endocapillary cellular proliferation obliterating capillary loops (× 400). Hematoxylin \& eosin. (B) Glomerulus demonstrating basement membrane double contours (× 600). Jones silver stain. (C) Electron microscopy of glomerulus with cellular obliteration of capillary loops and many electron dense deposits $(x$ $1,800)$. (D) Electron microscopy of capillary loop with large electron dense deposits $(\times 7,100)$.

it has been speculated that spontaneous resolution of severe AKI secondary to HCV-related MPGN may occur without intensive immunosuppressive therapy (intravenous and/or oral steroids, rituximab or cyclophosphamide \pm plasmapheresis) and/or HCV-directed anti-viral therapy [9], there are no well documented reports of this occurrence. Recurrent HCV-related MPGN in the kidney transplant is well documented $[10,11]$.

We report a recipient of a simultaneous liver-kidney transplant who developed significant AKI due to recurrent HCVrelated MPGN in the allograft. While the urinary findings and clinical course of her native kidney disease were consistent with HCV-related MPGN, its recurrence in the kidney transplant was associated with atypical urinary findings and unexpected spontaneous resolution of severe AKI. HCV infection was subsequently eradicated with an interferon-free anti-viral regimen.

\section{Case Report}

\section{Pre-transplant course}

A 45-year-old African American female was first evaluated in our institution for stage 3 chronic kidney disease and nephrotic syndrome. Her past medical history included long-standing hypertension, liver cirrhosis (initially diagnosed by imaging and subsequently confirmed by liver biopsy) and HCV genotype $1 \mathrm{~b}$ infection of at least 11 years duration (with negative HBV-surface antigen and core antibody) and past tobacco/alcohol/nasal cocaine use which she quit several years earlier. Patient had received a short course of $\alpha$-interferon ( $\alpha$-INF) and ribavirin 8 years earlier, but treatment was discontinued due to side effects. She had required abdominal paracentesis for refractory ascites on a few occasions, but there was no history of hepatic encephalopathy or variceal bleeding. At the time of initial evaluation, there was marked ascites and severe bilateral/symmetrical pitting lower extremity edema up to the mid-thighs, proteinuria and microhematuria (10 - $20 \mathrm{RBCs} /$ high power field (hpf)) and no casts. Serum creatinine level was $1.9 \mathrm{mg} / \mathrm{dL}$ with estimated GFR of $36 \mathrm{~mL} / \mathrm{min} / 1.73 \mathrm{~m}^{2}$. Urine protein/creatinine ratio was $3,500 \mathrm{mg} / \mathrm{g}$. RF test was positive $(96 \mathrm{IU} / \mathrm{mL}$, normal $<15 \mathrm{IU} / \mathrm{mL})$, but the serum cryoglobulin screen was reported as negative. Serum complement levels were low: C3 $57 \mathrm{mg} / \mathrm{dL}$ and C4 $<2 \mathrm{mg} / \mathrm{dL}$. Anti-nuclear, anti-neutrophil cytoplasmic, Sjogren's and anti-glomerular basement membrane antibodies, and liver autoantibody screen were negative. Except for serum albumin of $2.2 \mathrm{~g} / \mathrm{dL}$ and total 

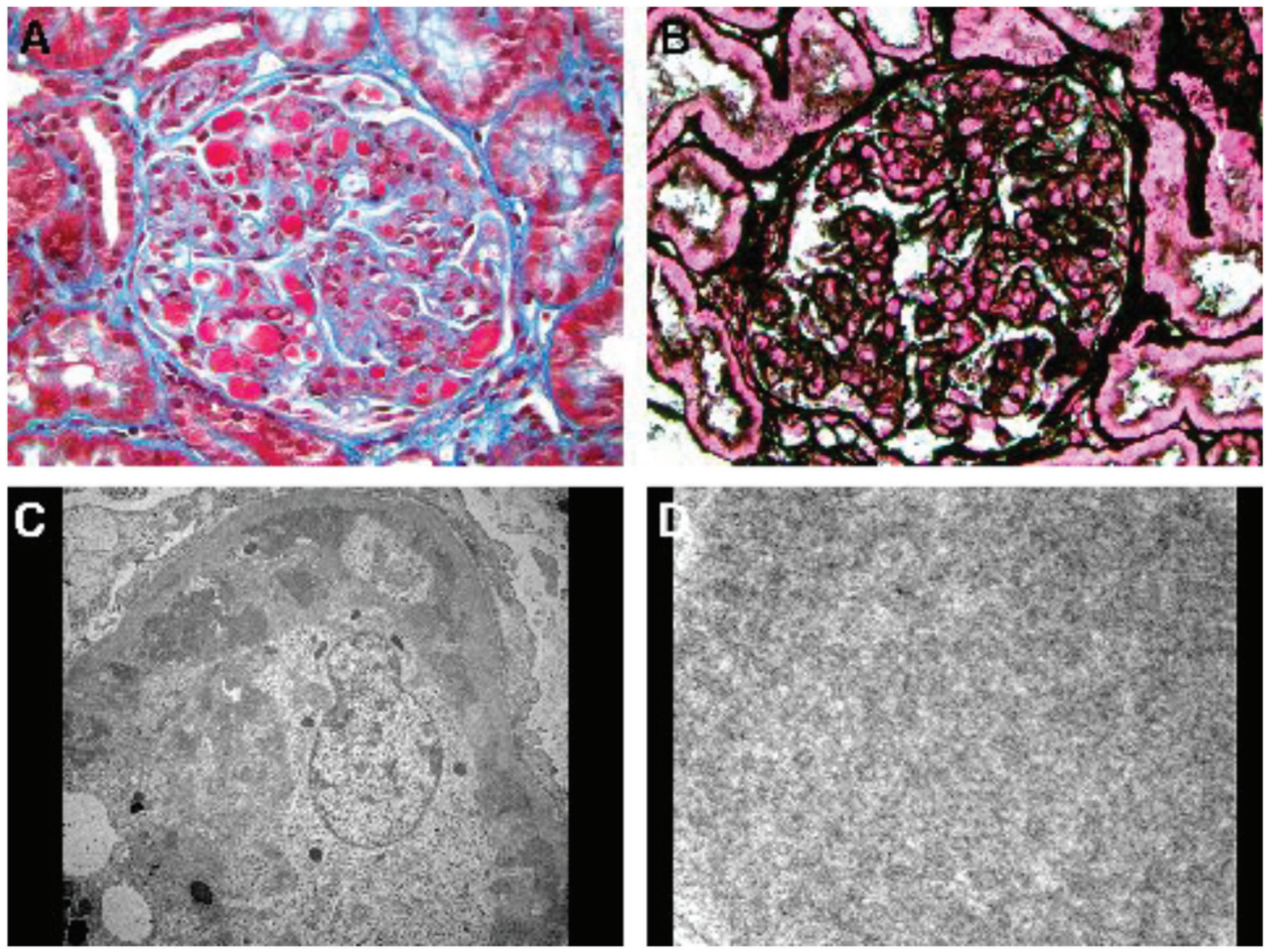

Figure 2. (A) Glomerulus exhibiting many prominent (red) cryoglobulin deposits in capillary loops ( $\times 400)$. Trichrome stain. (B) Glomerulus exhibiting basement membrane double contours $(\times 400)$. Jones silver stain. (C) Electron microscopy of a glomerular capillary loops obliterated by cells and cryoglobulin deposits $(\times 7,100)$. (D) Electron microscopy of cryoglobulin deposits demonstrating tubular structure $(\times 89,000)$.

protein of $4.5 \mathrm{~g} / \mathrm{dL}$, rest of the liver function tests (including prothrombin time) were normal. Serum HCV-RNA by PCR was $432,657 \mathrm{IU} / \mathrm{mL}$. Immunofixation revealed a small IgMkappa monoclonal protein $(0.2 \mathrm{~g} / \mathrm{dL})$ in the mid-gamma region in the serum. Renal ultrasound showed kidneys of normal size and echogenicity without hydronephrosis. Native kidney biopsy revealed immune complex-mediated MPGN with extensive subendothelial and some mesangial electron dense deposits with mesangial interposition and glomerular capillary wall duplication, consistent with HCV-associated MPGN (Fig. 1). Direct immunofluorescence was positive for IgG, IgA, IgM, $\mathrm{C} 3$, and kappa and lambda light chains. However, the electron dense deposits did not have the characteristic crystalline/tubular structure of cryoglobulins. Within 1 month after the biopsy, she was started on maintenance hemodialysis due to diureticresistant anasarca and rapid decline in kidney function. She received a simultaneous liver-kidney transplant after 3 years on hemodialysis.

\section{Post-transplant course}

Immunosuppression consisted of rabbit anti-thymocyte globu- lin induction (two doses), prednisone $30 \mathrm{mg} /$ day tapered to 5 $\mathrm{mg}$ /day at 2 months, mycophenolate mofetil (MMF) $500 \mathrm{mg}$ twice a day and of tacrolimus (target trough levels $8-12 \mathrm{ng} /$ $\mathrm{mL}$ in first 3 months, $5-8 \mathrm{ng} / \mathrm{mL}$ thereafter).

She maintained normal liver function (except for variable elevation of serum alkaline phosphatase level up to $416 \mathrm{IU} / \mathrm{L}$, normal $<120 \mathrm{IU} / \mathrm{L}$ ), probably related to recurrent HCV-hepatitis as documented by liver biopsy 2 months post-transplant, serum albumin between 3.0 and $3.7 \mathrm{~g} / \mathrm{dL}$ and excellent renal function (serum creatinine $0.64-1.02 \mathrm{mg} / \mathrm{dL}$ ) with normal urinalysis over the next 10 months.

She was hospitalized 10 months post-transplant for AKI (serum creatinine $2.26 \mathrm{mg} / \mathrm{dL}$ ) and hyperkalemia (6.3 $\mathrm{mmol} / \mathrm{L}$ ) due to elevated tacrolimus level (> $30 \mathrm{ng} / \mathrm{mL}$ ). With tacrolimus dose reduction serum creatinine returned to $1.1 \mathrm{mg} /$ $\mathrm{dL}$ and hyperkalemia resolved. She received furosemide intermittently during the first 6 days of hospitalization for treatment of hyperkalemia and lower extremity edema. On hospital day 6 , serum creatinine started to rise again peaking at $2.58 \mathrm{mg} /$ $\mathrm{dL}$ on day 15 despite acceptable tacrolimus trough levels $(4.8$ $-6.1 \mathrm{ng} / \mathrm{mL}$ ), and ultrasonography of the allograft was normal. Transplant kidney biopsy was performed on hospital day 15 for suspected acute rejection. Urinalysis immediately before 
biopsy revealed $30 \mathrm{mg} / \mathrm{dL}$ of albumin, no blood, $1 \mathrm{RBC} / \mathrm{hpf}$ and no casts. The biopsy unexpectedly revealed cryoglobulinemic glomerulonephritis with multiple glomerular capillaries filled with aggregates of electron dense material of variably coarse granular and poorly formed tubular ultrastructural appearance (Fig. 2). Similar electron dense material was also noted in subendothelial and mesangial locations. There was increased glomerular cellularity, podocyte effacement and capillary wall duplication, all in segmental distribution. There were no crescents. Direct immunofluorescence stains were positive for IgG, IgA, IgM, and both kappa and lambda light chains.

Tests done post-biopsy revealed: C3 complement $49 \mathrm{mg} /$ $\mathrm{dL}, \mathrm{C} 4$ complement $<2 \mathrm{mg} / \mathrm{dL}, \mathrm{RF}$ positive and positive cryoglobulin screen (type 2 cryoglobulins $25.6 \mathrm{mg} / \mathrm{dL}$ ) with monoclonal IgM-kappa and polyclonal IgG. HCV-RNA by PCR was markedly elevated $(41,073,547 \mathrm{IU} / \mathrm{mL})$ compared to pretransplant values.

Since serum creatinine had almost tripled from baseline, plasmapheresis and rituximab therapy were considered for severe recurrence of HCV-related cryoglobulinemic MPGN. However, serum creatinine started to improve spontaneously and unexpectedly the day after the kidney biopsy without any change in her maintenance immunosuppression or other additional therapy, and returned to baseline $(0.94 \mathrm{mg} / \mathrm{dL}) 10$ days later.

In the hopes of preventing progressive liver damage and recurrence of HCV-related renal problems, anti-HCV therapy was started with oral sofosbuvir $400 \mathrm{mg}$ daily and ribavirin $400 \mathrm{mg}$ twice a day 3 months later. After 4 months of this therapy, serum HCV-RNA is undetectable, serum creatinine is $1.04 \mathrm{mg} / \mathrm{dL}$, urinalysis shows only trace proteinuria and normal sediment, liver function tests are normal (including serum alkaline phosphatase level), serum albumin level is $4.2 \mathrm{~g} / \mathrm{dL}$, serum C3 (108 mg/dL) and C4 (27 mg/dL) complements are normal, $\mathrm{RF}<15 \mathrm{IU} / \mathrm{mL}$, and cryoglobulin screen are negative, with no apparent adverse effects from anti-viral therapy. Current immunosuppression consists of MMF $500 \mathrm{mg}$ twice 1 day and tacrolimus (target trough levels $5-8 \mathrm{ng} / \mathrm{mL}$ ).

\section{Discussion}

Recurrent HCV-related hepatitis and glomerular disease in the liver and kidney transplants, respectively, are well documented [12]. However, our patient had a very atypical clinical presentation and clinical course. While the presentation of her native kidney disease was consistent with the known clinical features of HCV-related MPGN, that of the recurrent disease in the kidney transplant was not in two respects: the findings on urinalysis (minimal proteinuria, no blood and only $1 \mathrm{RBC} / \mathrm{hpf}$ with no casts) did not suggest MPGN. Secondly, the recurrent renal disease was clinically severe with tripling of the serum creatinine level and the rapid and complete resolution of AKI of this severity without additional immunosuppression, plasmapheresis or anti-viral therapy was surprising. To our knowledge, spontaneous resolution of AKI due to cryglobulinemic MPGN in native kidneys or renal allografts has not been reported.

Treatment of cryoglobulinemic MPGN depends on sever- ity of kidney involvement. If kidney involvement is characterized by significant proteinuria with stable and good kidney function, anti-viral therapy alone is recommended. However, the established $\alpha$-INF-based anti-viral regimen carries with it the risk of precipitating acute rejection of the transplanted organ. Montalbano et al have reported successful treatment of cryoglobulinemic syndrome with INF and ribavirin in a simultaneous liver-kidney transplant recipient with only a small drop in creatinine clearance from $74 \mathrm{~mL} / \mathrm{min}$ pretreatment to $52 \mathrm{~mL} / \mathrm{min}$ during the treatment [12]. The authors speculated that this successful outcome might have been due to the known ability of the liver graft to protect the kidney graft against rejection.

If HCV-related MPGN is associated with significant AKI in addition to proteinuria, immediate institution of aggressive immunosuppression with high dose intravenous and/or oral corticosteroids + rituximab (or cyclophosphamide) \pm plasmapheresis is recommended (for as short a period as possible to minimize risk of rapid increase in HCV load) [13]. We considered such therapy but renal function unexpectedly recovered without any intervention. The cause of sudden recurrence and spontaneous resolution of HCV-related MPGN in our patient is unclear.

Since we do not have follow-up kidney biopsy documentation that the resolution of AKI coincided with resolution of MPGN, it may be argued that the recurrent MPGN was only histological, and AKI was unrelated to MPGN. However, we believe that the steady increase in the serum creatinine level over a 9-day period during which tacrolimus levels were not elevated, and there was no evidence of pre-renal azotemia or obstructive uropathy, makes recurrent MPGN the most likely cause of AKI. In our opinion, re-biopsy to document disappearance of MPGN was not justified given the complete resolution of her AKI.

Since the HCV load had increased (as expected) posttransplant, we wished to prevent further recurrences of renal allograft problems and also prevent progressive liver allograft injury by using anti-HCV therapy. The availability of $\alpha$-INFfree newer anti-viral regimens made this possible without risking acute rejection. The response of our patient to sofosbuvir + ribavirin regimen has been gratifying with disappearance of HCV viremia and cryoglobulinemia, stable/excellent renal transplant function, normal urinalysis, normalization of serum alkaline phosphatase level (possibly reflecting improvement in recurrent $\mathrm{HCV}$ hepatitis in the liver graft) and resolution of hypocomplementemia.

In summary, our patient illustrates the following interesting features: 1) HCV-related MPGN may recur in the kidney transplant without typical urinary findings; 2) the resultant (even severe) AKI may occasionally resolve spontaneously without treatment; and 3) the successful outcomes that can be achieved with the newer $\alpha$-INF-free anti-HCV regimens.

\section{Conflict of Interest}

The authors report no conflicts of interest. The authors alone are responsible for the content and writing of the paper. 


\section{Abbreviations}

HCV: hepatitis C virus; MPGN: membranoproliferative glomerulonephritis; AKI: acute kidney injury; MMF: mycophenolate mofetil; $\alpha$-INF: $\alpha$-interferon; hpf: high power field

\section{References}

1. Brunkhorst R, Kliem V, Koch KM. Recurrence of membranoproliferative glomerulonephritis after renal transplantation in a patient with chronic hepatitis C. Nephron. 1996;72(3):465-467.

2. Johnson RJ, Gretch DR, Yamabe H, Hart J, Bacchi CE, Hartwell P, Couser WG, et al. Membranoproliferative glomerulonephritis associated with hepatitis $\mathrm{C}$ virus infection. N Engl J Med. 1993;328(7):465-470.

3. Ferri C, Longombardo G, La Civita L, Bombardieri S, Greco F, Highfield P, Corbishley T. Hepatitis C virus, autoimmune liver disease and cryoglobulinaemic hepatitis. J Hepatol. 1992;16(1-2):242-243.

4. Agnello V, Chung RT, Kaplan LM. A role for hepatitis $\mathrm{C}$ virus infection in type II cryoglobulinemia. N Engl J Med. 1992;327(21):1490-1495.

5. Sethi S, Fervenza FC. Membranoproliferative glomerulonephritis--a new look at an old entity. N Engl J Med. 2012;366(12):1119-1131.

6. Wintrobe M, Buell M. Hyperproteinemia associated with multiple myeloma: with report of a case in which an extraordinary hyperproteinemia was associated with thrombosis of the retinal veins and symptoms suggesting Raynaud's disease. Bull Hopkins Hosp. 1933;52(2):156164.

7. Lerner AB, Watson CJ. Studies of cryoglobulins; unusual purpura associated with the presence of a high concentration of cryoglobulin (cold precipitable serum globulin). Am J Med Sci. 1947;214(4):410-415.

8. Motyckova G, Murali M. Laboratory testing for cryoglobulins. Am J Hematol. 2011;86(6):500-502.

9. Tarantino A, Campise M, Banfi G, Confalonieri R, Bucci A, Montoli A, Colasanti G, et al. Long-term predictors of survival in essential mixed cryoglobulinemic glomerulonephritis. Kidney Int. 1995;47(2):618-623.

10. Hiesse C, Bastuji-Garin S, Santelli G, Moulin B, Cantarovich M, Lantz O, Charpentier B, et al. Recurrent essential mixed cryoglobulinemia in renal allografts. Report of two cases and review of the literature. Am J Nephrol. 1989;9(2):150-154.

11. Tarantino A, Moroni G, Banfi G, Manzoni C, Segagni S, Ponticelli C. Renal replacement therapy in cryoglobulinaemic nephritis. Nephrol Dial Transplant. 1994;9(10):1426-1430.

12. Montalbano M, Pasulo L, Sonzogni A, Remuzzi G, Colledan M, Strazzabosco M. Treatment with pegylated interferon and ribavirin for hepatitis $\mathrm{C}$ virus-associated severe cryoglobulinemia in a liver/kidney transplant recipient. J Clin Gastroenterol. 2007;41(2):216-220.

13. KDIGO Clinical Practice Guideline for Glomerulonephritis. Chapter 9: Infection-related glomerulonephritis. Kidney Int. (supplement) 2012;2:200-208. 